ISSN 0103-5150

Fisioter. Mov., Curitiba, v. 24, n. 2, p. 337-343, abr./jun. 2011

Licenciado sob uma Licença Creative Commons

\title{
Análise do equilíbrio em pacientes hemiparéticos após o treino com o programa Wii Fit
}

\author{
Balance analysis in hemiparetics patients after training with \\ Wii Fit program
}

\section{Luciana Barcala $^{[a]}$, Fernanda Colella ${ }^{[b]}$, Maria Carolina Araujo ${ }^{[c]}$, Afonso Shiguemi Inoue Salgado ${ }^{[\mathrm{d}]}$, Claudia Santos Oliveira ${ }^{[e]}$}

[a] Mestranda em Ciências da Reabilitação da Universidade Nove de Julho (UNINOVE), São Paulo, SP - Brasil, e-mail: lubarcala@hotmail.com

[b] Discente do curso de Fisioterapia da Universidade Nove de Julho (UNINOVE), São Paulo, SP - Brasil, e-mail: fernandacolella@gmail.com

[c] Discente do curso de Fisioterapia da Universidade Nove de Julho (UNINOVE), São Paulo, SP - Brasil, e-mail: carol.maraujo@uol.com.br

[d] Fisioterapeuta, Mestre pela Faculdade de Medicina da UNESP, Doutorando em Engenharia Biomédica pela Unicastelo, coordenador da Pós-Graduação em Terapia Manual da CESUMAR, Londrina, PR - Brasil, e-mail: afonsosisalgado@yahoo.com.br

[e] Docente do Programa de Mestrado em Ciências da Reabilitação da Universidade Nove de Julho (UNINOVE), São Paulo, SP Brasil, e-mail: csantos@uninove.br

\section{Resumo}

Objetivo: A hemiparesia é um comprometimento parcial do hemicorpo que altera o equilíbrio, sendo este essencial para as atividades funcionais. Objetivo: Avaliar o equilíbrio em pacientes hemiparéticos submetidos ao treino de equilíbrio com o programa Wii Fit, que atuou como um recurso de biofeedback visual. Método: Foram selecionados 12 pacientes hemiparéticos pós AVE, 5 do sexo masculino e 7 do sexo feminino, com idade média de $58 \pm 12,57$ anos, divididos aleatoriamente em dois grupos. Um deles realizou a fisioterapia convencional (GC) pelo período de uma hora, o outro realizou por trinta minutos e mais trinta minutos de treino de equilíbrio com auxílio do Wii Fit (GW), duas vezes por semana durante cinco semanas, completando dez sessões. 0 equilíbrio foi avaliado antes e após as intervenções, por meio da aplicação da Escala de Equilíbrio de Berg (EEB) e pela estabilometria, que mensura a oscilação do centro de pressão (COP), nos eixos ântero-posterior (AP) e médio-lateral (ML), por uma plataforma de pressão em duas condições: 
de olhos abertos (OA) e olhos fechados (OF). Resultados: De acordo com a EEB, os pacientes, tanto do GC quanto o do GW, obtiveram maior controle do equilíbrio estático e dinâmico. Na avaliação do COP no eixo ML, os indivíduos do GC e do GW tiveram diminuição na oscilação ML após a intervenção proposta para cada grupo, nas condições de OA e OF. No eixo AP do COP, o GC não teve diminuição na oscilação AP de OA e OF, e o GW apresentou diminuição na oscilação AP de OA e OF. Conclusão: 0 presente estudo demonstra que a fisioterapia associada ao treino de equilíbrio com o Wii Fit apresenta resultados significantes na reabilitação dos indivíduos hemiparéticos, obtendo, assim, mais um recurso terapêutico na fisioterapia.

Palavras-chave: Equilíbrio postural. Acidente vascular cerebral. Retroalimentação sensorial.

\section{Abstract}

Introduction: TThe hemiparesis is a partial compromise of the hemisphere that alters the balance, which is essential for the functional activities. Objective: To evaluate the balance in hemiparetic patients undergoing balance training with the Wii Fit (WG) program that served as a resource for visual biofeedback. Method: We studied 12 hemiparetic patients after stroke, 5 males and 7 females, mean age $58 \pm 12.57$ years old, were randomly divided into two groups. One group performed conventional physiotherapy (CP) for a period of one hour, the other group performed the traditional physical therapy for thirty minutes and thirty more minutes of balance training with the help of the Wii Fit $(W G)$, twice weekly for five weeks, completing ten sessions. Balance was assessed before and after intervention by the application of the Berg Balance Scale (BBS) and stabilometry which measures the oscillation of pressure center (COP) in anteroposterior axis (AP) and mediolateral (ML), a pressure platform in two conditions, with eyes opened (EO) and eyes closed (EC). Results: According to the BSE, patients from both the CG and the WG, have gained more control of static and dynamic balance. In assessing the COP in ML axis, the subjects of the CG and WG ML oscillation had decreased after the proposed intervention for each group, under the EO and EC. In the AP axis of the COP, the $C G$ had no decrease in the AP oscillation of EO and EC, and WG had a decrease in the AP oscillation in $O A$ and $O F$. Conclusion: This study shows that physiotherapy combined with balance training with Wii Fit presents significant results in the rehabilitation of hemiparetic subjects, thereby obtaining another therapeutic option in physiotherapy.

Keywords: Postural balance. Stroke. Feedback sensory.

\section{Introdução}

A Organização Mundial da Saúde define Acidente Vascular Encefálico (AVE) como um episódio de início rápido e abrupto, de origem vascular, que reflete em uma perturbação focal ou generalizada da função encefálica, excluindo deficiências isoladas e persistindo por mais de 24 horas. 0 diagnóstico é confirmado por exames clínicos, tomografia computadorizada e ressonância magnética (1).

Os principais fatores de risco do AVE incluem hipertensão arterial sistólica ou diastólica, hipercolesterolemia, tabagismo, diabetes mellitus, consumo elevado de álcool, sedentarismo, estresse e uso de anticoncepcionais orais, sendo evidente a falta de adesão ao tratamento e/ou a modificação de hábitos $(2,3)$.

Cerca de 50 a $70 \%$ dos indivíduos acometidos pelo AVE recuperam a independência funcional e, após seis meses, cerca de 50\% apresentam hemiparesia/hemiplegia, sendo a principal incapacidade crônica em adultos (4). Antigamente pensava-se que a recuperação seria dos primeiros três meses até um ano, porém, estudos mostram que as habilidades funcionais podem continuar melhorando com a frequencia na fisioterapia mesmo muitos anos após o AVE (5).

A deficiência motora determina uma seqüela, denominada hemiplegia, que consiste em um estado físico de um hemicorpo. É denominada plegia em virtude da paralisia total e, quando ocorre a paralisia motora parcial, é denominada paresia. Isso ocorre em razão da lesão do neurônio motor superior. A hemiplegia, ou paresia, é um dos maiores problemas enfrentados pelos pacientes que sofreram AVE $(6,7)$. Esse distúrbio pode causar redução de estabilidade, que é definida pela máxima distância que o indivíduo pode suportar o seu peso em alguma direção sem perda do equilíbrio (8).

Embora a hemiparesia seja o sinal mais óbvio do AVE, e também o principal objetivo da fisioterapia, 
outros sintomas estão incluídos na incapacidade do indivíduo, como a disfunção sensorial, a afasia ou a disartria, a visão e o comprometimento mental e intelectual (9).

A maior evidência do comprometimento hemiparético é a tendência em manter-se em uma posição assimétrica postural, com distribuição de peso menor sobre o hemicorpo parético. Essa assimetria e a dificuldade em transferir o peso para o lado afetado interferem na capacidade de manter o controle postural, impedindo a orientação e a estabilidade para realizar movimentos com o tronco e os membros (10).

0 equilíbrio é um processo complexo que depende da integração da visão, do sistema vestibular e sistema nervoso periférico, dos comandos centrais e das respostas neuromusculares e, particularmente, da força muscular e do tempo de reação. Para obter um melhor equilíbrio, um indivíduo procura manter o seu centro de massa corporal dentro dos seus limites de estabilidade, sendo esta determinada pela habilidade em controlar a postura sem alterar a base de suporte (11).

A postura estática humana é mantida por meio de um processo complexo envolvendo várias modalidades sensoriais. Juntos, esses sistemas interagem para a estabilização e a representação postural do corpo (12).

As alterações funcionais decorrentes do AVE variam de um indivíduo para outro e as alterações do equilíbrio apresentam índices elevados após o AVE, sendo necessário avaliar e elaborar as metas terapêuticas centradas na melhora do equilíbrio. 0 retreinamento do controle postural é essencial para a reabilitação $(8,13)$.

Em pacientes vítimas de AVE, a recuperação da habilidade para ficar em pé e andar é crítica, pois requer um complexo mecanismo do controle postural. Várias estratégias de tratamento são sugeridas para a manutenção do controle postural (14).

Após uma lesão cerebral, é indiscutível a importância da fisioterapia, pois propicia a reeducação dos movimentos e o equilíbrio postural. Para que a avaliação seja objetiva e útil, faz-se necessário o uso de medidas padronizadas e comprovadas, permitindo quantificar o objetivo fisioterapêutico. Os instrumentos mostram se ocorreram mudanças com o tempo. É importante que a medida seja validada e comprovada para estar de acordo com as variações no desempenho do indivíduo (9).

A Escala de Equilíbrio de Berg (EES) mede 14 itens do equilíbrio estático e dinâmico, atribuindo a cada item uma pontuação de 0 a 4 . A pontuação 0 representa inabilidade para completar um item e a pontuação 4 representa habilidade independente na tarefa. 0 escore total varia de 0 a 56 pontos $(9,15)$.

A estabilometria é uma técnica de avaliação do equilíbrio na postura ortostática que consiste na quantificação das oscilações ântero-posteriores e laterais do corpo enquanto o indivíduo permanece de pé sobre uma plataforma de pressão. Uma vez que a oscilação do centro de pressão (COP) é representada pelas oscilações posturais, o registro é feito pelo cálculo instantâneo da sua posição (coordenadas $\mathrm{x}, \mathrm{y}$ ), que corresponde à localização da resultante das pressões aplicadas na superfície em contato com os pés, que consiste na base de apoio. Para tal, normalmente são adotados períodos de curta duração, em torno de 30 segundos. A base de apoio é uma variável que, quando controlada, não exerce influência nos parâmetros do sinal (16).

0 treino por biofeedback visual, desenvolvido principalmente como uma ferramenta de aprendizagem motora e/ou capacidade de controle motor, tem evoluído muito na área da neurociência. Esse método envolve a constante autocorreção, durante uma série de tarefas motoras, pelo biofeedback visual, no qual as habilidades de planejamento motor e controle motor são continuamente estimuladas e benéficas na plasticidade neural (17).

0 estímulo visual relacionado com o equilíbrio tem sido demonstrado como um método eficaz para melhorar a postura simétrica após o AVE. Estudos mostram que pacientes submetidos ao treino visual melhoraram sua distribuição de peso nos membros e também obtiveram simetria no ortostatismo após fisioterapia convencional, com exercícios de equilíbrio em pé e de transferência de peso (18).

Alguns experimentos mostraram que a reabilitação de pacientes hemiparéticos, por meio do uso do biofeedback visual com sistemas de plataforma de pressão, melhora o equilíbrio estático, evidenciando que esse instrumento pode ser utilizado não apenas para avaliação quantitativa do equilíbrio, mas também como recurso para o treinamento do equilíbrio (11).

Os exercícios do programa Wii Fit da Nintendo ${ }^{\circledR}$ são um recurso a mais que tem sido utilizado nos centros de reabilitação, porém, poucos estudos comprovam a eficiência desse programa em pacientes neurológicos em reabilitação fisioterapêutica.

0 objetivo do estudo foi avaliar o equilíbrio em pacientes hemiparéticos antes e após a fisioterapia 
convencional e a fisioterapia associada ao treino de equilíbrio com o programa Wii Fit da Nintendo ${ }^{\circledR}$, que atuou como um recurso de biofeedback visual.

\section{Metodologia}

A pesquisa foi aprovada pelo Comitê de Ética em Pesquisa da Universidade Nove de Julho (Uninove) sob o número de protocolo 312133 de acordo com a resolução 196/96. O estudo foi realizado na Clínica Escola de Fisioterapia da Uninove, em São Paulo, Brasil. Trata-se de um ensaio clínico randomizado controlado, que foi realizado no período de janeiro a junho de 2010.

Foram avaliados os pacientes adultos hemiparéticos com diagnóstico de AVE, de ambos os sexos e em tratamento fisioterapêutico na Clínica da Uninove. Para inclusão no estudo, os pacientes concordaram e assinaram o Termo de Consentimento Livre e Esclarecido. Não foram incluídos os pacientes com doenças neurológicas não relacionadas ao AVE e os que não conseguiram interagir com os exercícios do programa Wii Fit. Participaram do estudo doze pacientes divididos aleatoriamente em dois grupos, sete do sexo feminino e cinco do sexo masculino, com idade média de $58 \pm 12,57$ anos, com tempo de lesão de $14 \pm 6,62$ meses.

A avaliação do equilíbrio foi realizada pela plataforma de pressão, da marca Fusyo, que dispõe de 2.300 sensores de pressão com precisão de $0,1 \mathrm{~mm}$ e aquisição de $40 \mathrm{~Hz}$. Essa plataforma analisa a oscilação anteroposterior (AP) e médio-lateral (ML). Na avaliação, os pacientes permanecem em posição ortostática sob a plataforma com a base irrestrita, os braços ao longo do corpo, durante 30 segundos, com os olhos abertos (OA) e, em seguida, com os olhos fechados (OF). As coletas foram realizadas antes e após o período de intervenção terapêutica.

Os pacientes incluídos no trabalho foram divididos em dois grupos. 0 primeiro grupo de pacientes submetidos ao tratamento com a fisioterapia convencional foi denominado de grupo convencional (GC). O segundo grupo, além da fisioterapia convencional, também participou do treinamento de equilíbrio com o auxílio do programa interativo Wii Fit, denominado grupo Wii (GW). Os pacientes foram avaliados pela EEB e pela plataforma de pressão (exame de estabilometria), a fim de verificar a evolução do equilíbrio e comparar as duas intervenções. Os dois grupos de pacientes foram submetidos ao tratamento de fisioterapia, completando dez sessões com duração de 60 minutos e com frequência de duas vezes por semana.

As sessões de treino de equilíbrio do GW foram individuais com 30 minutos de fisioterapia convencional e mais 30 minutos de exercícios de equilíbrio com o Wii Fit, mantendo a frequência de duas vezes por semana. Durante cada sessão, foram utilizados três tipos de exercícios: a plataforma, o peixe e a corda. 0 nível de dificuldade foi de acordo com a capacidade de interação de cada paciente com a atividade do programa Wii Fit. Cada exercício teve duração de 10 minutos, com intervalo de tempo para descanso entre cada um deles de acordo com o nível de condicionamento de cada paciente.

A amostra foi analisada estatisticamente pelos testes $\mathrm{t}$ pareado e não pareado com confiabilidade de $p<0,05$. Foram utilizados os software Instat $^{\circledR}$, para a análise estatística, e Minitab ${ }^{\circledR}$, para demonstração do resultado ilustrativo.

\section{Resultados}

A amostra foi composta por doze indivíduos e apresentou distribuição paramétrica.

0 Gráfico 1 mostra os valores em média e o desviopadrão da análise de equilíbrio de acordo com a pontuação da EEB, comparando o GC e o GW antes e após a intervenção. Pode-se observar que ambos os grupos apresentaram resultados significantes $(p<0,01)$ na aquisição do controle do equilíbrio estático e dinâmico.

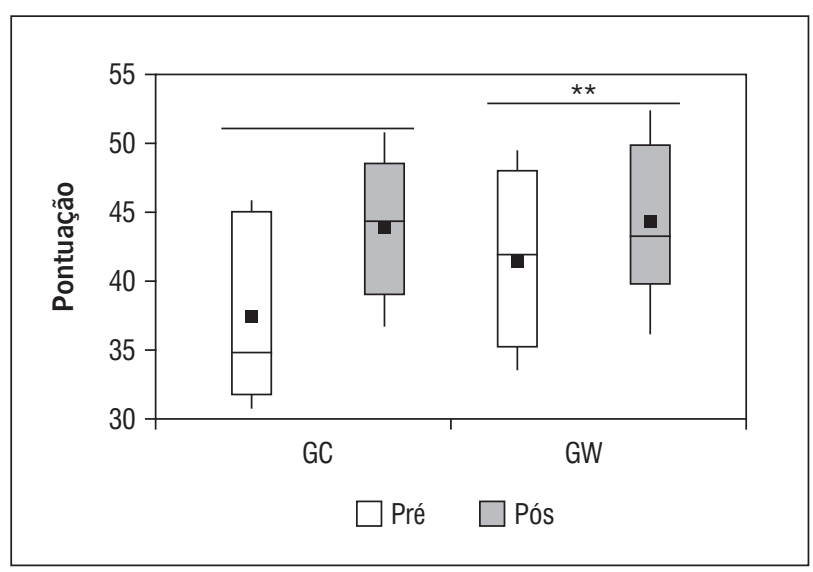

Gráfico 1 - Comparação (média e DP) do equilíbrio intragrupos mensurado pela Escala de Equilíbrio de Berg Legenda: ${ }^{*}=(p<0,01)$.

Fonte: Dados da pesquisa. 
Os valores do centro de oscilação corporal (COP) no eixo médio-lateral $(\mathrm{X})$ e ântero-posterior $(\mathrm{Y})$ foram definidos pelo protocolo de Winter (19), onde:

- Delta $X(\Delta X)=$ máxima oscilação do COP na direção X em mm = máxima COPX - mínimo COPX;

- Delta $\mathrm{Y}(\Delta \mathrm{Y})$ = máxima oscilação do COP na direção Y em mm = máxima COPY - mínimo COPY;

Na análise do COP no eixo X, médio-lateral (ML), ambos os grupos apresentaram diminuição nas oscilações ML, sendo os resultados mais significantes $(\mathrm{p}<0,01)$ nas condições de olhos abertos (OA) que em condições de olhos fechados (OF) $(\mathrm{p}<0,05)$. 0 Gráfico 2 mostra os valores médios de oscilação em mm, comparando os grupos pré e pós-intervenção nas condições de OA e OF.

$\mathrm{Na}$ análise do COP no eixo Y, ântero-posterior (A/P), o GC obteve diminuição nas oscilações AP em ambas as condições, mas esses valores não foram significantes ( $p>0,05)$. No GW, as oscilações AP foram diminuídas e obtiveram resultados estatisticamente significantes $(p<0,05)$. 0 Gráfico 3 demonstra a comparação entre os dois grupos em condições de $\mathrm{OA} \mathrm{e} \mathrm{OF}$.

Sendo assim, na análise do equilíbrio pela EEB e pelo COP ML, ambos os grupos apresentam os mesmos resultados significantes. No COP AP, somente o GW apresentou resultado. Isso mostra que o treino de equilíbrio associado ao programa de biofeedback visual (no caso Wii Fit) é um recurso em que o

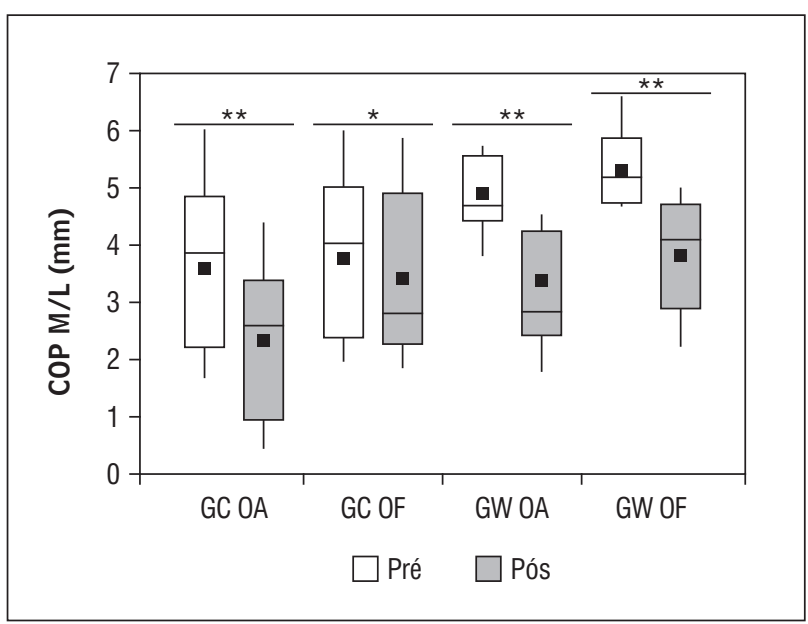

Gráfico 2 - Comparação (média e DP) do COP no eixo AP intragrupos nas condições de olhos abertos (OA) e olhos fechados (OF)

Legenda: ${ }^{*}=(p<0,05) ;{ }^{* *}=(p<0,01)$.

Fonte: Dados da pesquisa.

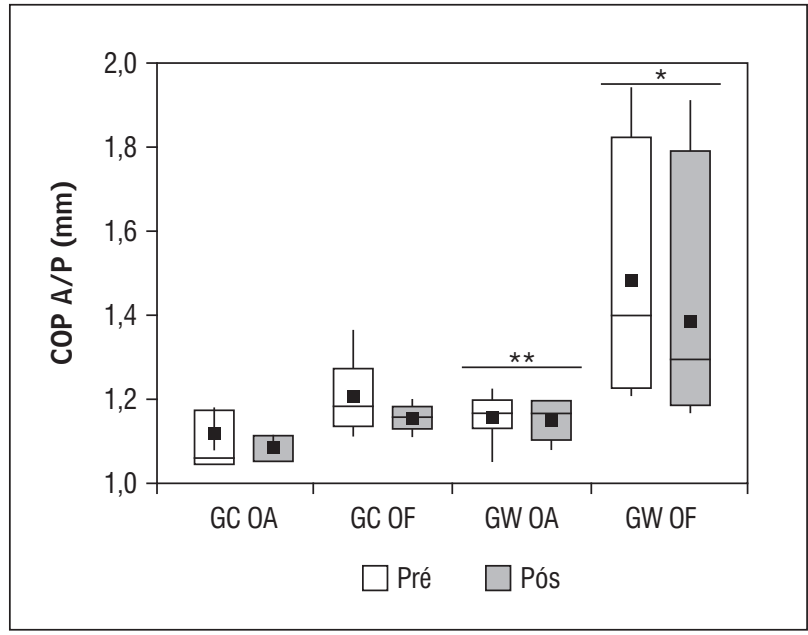

Gráfico 3 - Comparação (média e DP) do COP no eixo AP intragrupos nas condições de olhos abertos (OA) e olhos fechados (OF)

Legenda: ${ }^{*}=(p<0,05)$.

Fonte: Dados da pesquisa.

indivíduo poderá obter maior controle do equilíbrio dinâmico e diminuição das oscilações ML e AP do equilíbrio estático.

\section{Discussão}

A tecnologia virtual é uma realidade emergente com uma variedade de benefícios para os aspectos da avaliação de recuperação, tratamento e investigação. A investigação e a compreensão dessa tecnologia serão cruciais para a efetiva integração na reabilitação. Para isso é necessário um período de adaptação dos novos recursos tecnológicos, principalmente em indivíduos que não possuem a prática da tecnologia virtual $(20,21)$.

Um estudo realizado por Ching et al. (22) mostra que a reabilitação está evoluindo para fora da terapia tradicional, por isso há importância de estudos práticos para a comprovação da eficiência desse novo modelo de reabilitação.

A participação em atividades de lazer é um direito humano e fundamental para uma melhor qualidade de vida. Adultos com deficiência física muitas vezes experimentam poucas oportunidades de participação em atividades de lazer e de realidade virtual, sendo difícil aumentar suas possibilidades de interatividade pela falta de acessibilidade a essas atividades. Embora o uso das atividades virtuais na 
reabilitação tenha crescido nos últimos dez anos, poucas aplicações têm sido relatadas para as pessoas com deficiências neurológicas.

Não existem estudos sobre a melhora do equilíbrio em paciente com AVE após treinamento com o programa Wii Fit, porém, já foi realizado um estudo com 34 indivíduos com paralisia cerebral que participaram de jogos virtuais de 2 a 3 vezes por semana durante 12 semanas. Foi observada a interação agradável e bem-sucedida, com alto nível de interesse e de aprendizado. A facilidade de utilização e adaptabilidade torna-se uma opção viável (8).

0 acessório mais recente do Nintendo ${ }^{\circledR}$ Wii é o Balance Board, que exige do indivíduo uma mudança constante de alto desempenho na postura em pé e avalia a capacidade de controlar a estimulação ambiental, usando as mudanças corporais da postura em pé.

Estudo realizado com o Wii Balance Board verificou a funcionalidade da correção da postura em pé, isto é, ajustar ativamente a postura anormal em pé. Foi avaliado se duas pessoas com múltiplas deficiências seriam capazes de corrigir a sua postura ativa em pé de acordo com a estimulação. Os dados mostraram que ambos os participantes aumentaram significativamente a duração do tempo de manter a postura correta em pé, ativando assim o sistema de controle $(22,23)$.

Outro estudo (24), realizado no corrente ano, verifica a recuperação do déficit motor em membro superior em 20 pacientes com AVE e com idade de 18-85 anos, após a utilização de um ambiente virtual. 0 protocolo de reabilitação foi aplicado em oito sessões, cada uma de 60 minutos, sendo realizadas duas vezes semana. 0 resultado comprova a viabilidade, a segurança e a eficácia da realidade virtual por meio da tecnologia do programa Wii Fit na reabilitação da patologia.

De acordo com os estudos realizados por outros autores e o presente estudo, verifica-se a crescente abordagem da reabilitação virtual e a comprovação do Wii Fit no treino de equilíbrio.

\section{Conclusão}

Os resultados obtidos no presente estudo demonstram que o treino de equilíbrio, associado ao programa Wii Fit, proporciona resultados significantes na reabilitação fisioterapêutica, obtendo-se assim mais um recurso para o tratamento, sendo esse um recurso interativo e lúdico, podendo propiciar uma motivação a mais nas sessões de fisioterapia. A fisioterapia convencional mantém sua importância, pois o grupo submetido a esse tipo de tratamento também obtém resultados significantes na reabilitação. Graças ao aumento da utilização de ambientes virtuais auxiliando a reabilitação, torna-se importante a sua comprovação. Estudos futuros podem averiguar o aspecto da qualidade de vida nesses pacientes neurológicos submetidos a ambientes virtuais em sua reabilitação, assim como ao programa Wii Fit.

\section{Referências}

1. Duncan P, Studenski S, Richards L, Gollub S, Lai SM, Reker D, et. al. Randomized clinical trial therapeutic exercise in subacute stroke. Stroke. 2003;34(9): 2173-80.

2. McManus JA, Craig AMC, Langhorne P, Ellis G. Does behaviour modification affect post-stroke risk factor control? Three-year follow-up of a randomized controlled trial. Clinical Rehabil. 2009;23(2):99-105.

3. Van de Port IGI, Wevers L, Roelse H, Kats LV, Lindeman E, Kwakkel G. Cost-effectiveness of a structured progressive task-oriented circuit class training programme to enhance walking competency after stroke: the protocol of the FIT-Stroke trial. BMC Neurol. 2009;9(43):1-9.

4. Marucci FCI, Cardoso NS, Berteli KS, Garanhani MR, Cardoso JR. Alterações eletromiográficas dos músculos do tronco de pacientes com hemiparesia após acidente vascular encefálico. Arq Neuropsiquiatr. 2007;65(3-b):900-5.

5. Dean CM, Rissel C, Sharkey M, Sherrington C, Cumming RG, Barker RN, et al. Exercise intervention to prevent falls and enhance mobility in community dwellers after stokes. BMC Neurol. 2009;9:38.

6. Rezende FB, Viana CAP, Faria JLC. Análise da hiper-extensão de joelho em pacientes hemiparéticos usando órtese para neutralização da flexão plantar. Rev Neurociências. 2006;14(3):140-3.

7. Ottoboni C, Fontes SV, Fukujima MM. Estudo comparativo entre marcha normal e a de pacientes hemiparéticos por acidente vascular encefálico: aspectos biomecânicos. Rev Neurociências. 2002;10(1):10-6. 
8. Torriani C, Queiroz SS, Sakakura MT, Zicati M, Volpini AF, Trindade AA, et al. Estudo comparativo de equilíbrio de pacientes com disfunção cerebelar e com seqüelas de acidente vascular encefálico. RBPS. 2005; 18(3):157-61.

9. Carvalho AC, Vanderlei LC, Bofi TC, Pereira JDAS, Nawa VA. Projeto hemiplegia: um modelo de fisioterapia em grupo para hemiplégicos crônicos. Arq Ciênc Saúde. 2007;14(3):161-8.

10. Ikai T, Kamikubo T, Nishi M, Miyani S. Dynamic postural control in patients with hemiparesis. Am J Phys Med Rehabil. 2003;82(6):463-9.

11. Overstall PW. The use of balance training in elderly people with falls. Clinical Geron. 2003;13(2)153-61.

12. Pontelli TEGS, Pontes-Neto OM, Colafêmina JF, de Araújo DB, Santos AC, Leite JP. Controle postural na síndrome de Pusher: influência dos canais semicirculares laterais. Rev Bras Otorrinolaringol. 2005; 71(4):448-52.

13. Furini V, Oréfice RSR, Laraia SEM, Soares NC. Avaliação do equilíbrio estático orientado pelo desempenho de portadores de acidente vascular encefálico. Ter Man. 2008;6(27):303-6.

14. Garland SJ, Willems DA, Ivanova TD, Miller KJ. Recovery of standing balance and functional mobility after stroke. Arch Phys Med Rehabil. 2003;84(12):1753-9.

15. Conradsson M, Lundin-Olsson L, Lindelöf N, Littbrand $\mathrm{H}$, Malmqvist L, Gustafson Y, et al. Berg balance scale: intrarater test retest reliability among older people dependent in activities of daily living and living in residential care facilities. Phys Ther. 2007; 87(9):1155-63.

16. Chiari L, Rocchi L, Cappello A. Stabilometric parameters are affected by anthropometry and foot placement. Clin Biomech. 2002;17(9-10):666-77.

17. Cho SH, Shin HK, Kwon YH, Lee MY, Lee YH, Lee CH, et al. Cortical activation changes induced by visual biofeedback tracking training in chronic stroke patients. NeuroRehabilitation. 2007;22(2):77-84.
18. Walker C, Brouwer BJ, Culham EG. Use of visual feedback in retraining balance following acute stroke. Physical therapy. 2000;80(9):886-95.

19. Winter DA. Human balance and posture control during standing and walking. Gait Post. 1995;3:193-214.

20. Srivastava A, Taly AB, Gupta A, Kumar S, Murali T. Poststroke balance training: role of force platform with visual feedback technique. J Neurol Sci. 2009;287(1-2): 89-93.

21. Saposnik G, Mamdani M, Bayley M, Thorpe KE, Hall J, Cohen LG. Effectiveness of virtual reality exercises in stroke rehabilitation (EVREST): rationale, design, and protocol of a pilot randomized clinical trial assessing the Wii gaming system. Int J Stroke. 2010; 5(1):47-51.

22. Ching HS, Man LC, Ching TS. A limb action detector enabling people with multiple disabilities to control environmental stimulation through limb action with a Nintendo Wii remote controller. Research in Developmental Disabilities. 2010;31(5):1047-53.

23. Shih CH, Shih CT, Chu CL. Assisting people with multiple disabilities actively correct abnormal standing posture with a Nintendo Wii Balance Board through controlling environmental stimulation. Res Dev Disabil. 2010;31(4):936-42.

24. Yalon-Chamovitz S, Weiss PL. Virtual reality as a leisure activity for young adults with physical and intellectual disabilities. Res Dev Disabil. 2008;29(3): 273-87.
Recebido: 07/10/2010

Received: 10/07/2010

Aprovado: 19/03/2011

Approved: 03/19/2011 